\title{
Binocular coordination during smooth pursuit in dyslexia: a multiple case study
}

\author{
Qing Yang \\ IRIS laboratory, FRE 3154, CNRS
}

\author{
Maria-Pia Bucci \\ IRIS laboratory, FRE 3154, CNRS
}

\author{
Marine Vernet \\ IRIS laboratory, FRE 3154, CNRS \\ Zoi Kapoula \\ IRIS laboratory, FRE 3154, CNRS
}

\begin{abstract}
Smooth pursuit (SP) was explored in dyslexics and non-dyslexics. Dyslexic children show similar gain of SP, and number and amplitude of catch-up saccades (CUS) as non-dyslexic children. The quality of binocular coordination is good for both groups; the only significant exception is for pursuit to the right for both smooth phase and CUS; dyslexics show higher disconjugacy. Decrement of binocular control during rightward pursuit only could reflect immaturity of oculomotor learning mechanisms needed to optimize binocular coordination for all directions. Yet, these observations need to be confirmed in a larger population including older children and compared with other populations, e.g. with right-to-left reading.
\end{abstract}

\footnotetext{
Keywords: Smooth pursuit, Developmental dyslexia, Binocular coordination, Catch-up saccades
}

\section{Introduction}

Dyslexia is a selective impairment of reading and spelling abilities without a deficit in general intelligence. Dyslexia is commonly attributed to a phonological deficits (e.g., Ramus, Rosen, Dakin, Day, Castellote, White \& Frith, 2003). However, a mild cerebellum dysfunction (Nicolson, Fawcett \& Dean, 2001) and specific visual problems, such as a defective magnocellular system, have also been discussed as possible causal factors (Stein, 2001). The latter theory has been supported by many studies showing abnormal eye movements during reading in dyslexics, such as longer fixations, smaller saccade amplitudes and a higher percentage of regression, i.e. backward saccades (Pavlidis, 1981). Also abnormalities of other types of eye movements are reported: inaccurate saccades (Biscaldi, Gezeck \& Stuhr, 1998), longer latency for saccades or vergence eye movements (Bucci, Bremond-Gignac \& Kapoula, 2008a), higher rates of express latencies for saccades (Biscaldi, Fischer \& Aiple, 1994) and for divergence (Bucci et al., 2008a), more frequent saccadic intrusions during smooth pursuit (Eden, Stein, Wood \& Wood, 1994), binocular instability (Stein, Richardson \& Fowler, 2000) and disordered vergence control (Stein, Riddell \& Fowler, 1988).
Since the magnocellular system dominates the visual guidance of eye movements by the posterior parietal cortex - PPC (Stein, 2001), cerebellum (Rae, Karmiloff-Smith, Lee, Dixon, Grant, Blamire, Thompson, Styles \& Radda, 1998) and superior colliculi - SC(Sparks \& Jay, 1986), slight dysfunction may affect the most vulnerable oculomotor control system, e.g. the binocular control system for any type of eye movements. The unstable binocular control of dyslexics probably explains the unstable visual perceptions that they experience. Cornelissen, Hansen, Hutton, Evangelinou and Stein (1998) reported that letters seem to move around, merge, flip and jump over each other. Such subjective reports describe the kind of fluctuating diplopia that might result from unstable binocular motor control and fixation. Children with such unsteady eyes tend to confuse and disarrange letters when attempting to read, so that they often misread real words as nonsense words (Cornelissen, Bradley, Fowler \& Stein, 1991). Recently, Bucci, BremondGignac and Kapoula (2008b) quantified binocular coordination during saccades to single word or target and reported decreased coordination in dyslexics relative to age-matched non-dyslexic children; similar observation was made by Kapoula, Bucci, Ganem, Poncet, Daunys and Bremond-Gignac (2008) in dyslexics for saccades and fixations during free exploration of paintings. The latter results indicate an intrinsic ocular motor deficiency 
independently from the reading process; deficiency could be related to immaturity of the normal ocular motor learning mechanism, upon which is based normal binocular motor development. In addition to cerebellum, the posterior parietal cortex could be instrumental for such binocular motor learning; a central interaction between saccade and vergence commands could be the mechanism by which binocular motor control improves (Vernet, Yang, Daunys, Orssaud, Eggert \& Kapoula, 2008).

Small saccades can also appear during (SP), i.e. catch-up saccades (CUS). Black, Collins, De Roach and Zubrick (1984) reported high rates of CUS during SP in dyslexic. In addition, Eden et al. (1994) found poor SP in dyslexics, particularly when pursuing a target moving from left to right. Although the mechanisms that controls the both CUS and SP are still poorly understood, common structures seem to be involved in their control. For example, motor or position error signal in the superior colliculus could be shared by the saccadic and smooth system (Krauzlis, Basso \& Wurtz, 2000). Lesions of the oculomotor cerebellar vermis affect both saccades and smooth pursuit (Takagi, Zee \& Tamargo, 2000). At the cortical level, there is anatomical evidence for connections between structures containing subregions for saccades and pursuit (Tian \& Lynch, 1996).

The goal of this study is to examine the quality of binocular coordination during CUS and pursuit phase of SP in dyslexic and non-dyslexic children. In both instances the eyes should move together so that the target stays on both foveae.

\section{Methods}

\section{Participants}

Seven dyslexic children were recruited from a college in Paris with classes specialized for dyslexia. Before entering these special classes, all children had been diagnosed either at a pediatric hospital or other medical centers. In the year of the present study, they underwent extensive examination including neurological/psychological and phonological tests, evaluation for the speed of reading, text comprehension and the capacity of reading word/pseudowords by the L2MA battery (ChevrieMuller, Simon \& Fournier, 1997). This is the standard test developed by the Applied Psychology Center of Paris and used everywhere in France. Inclusion criteria were: scores on L2MA test below two standard deviations but normal mean intelligence quotient (IQ, evaluated with WISC III), i.e. between 90 and 120. The mean age of the dyslexic children was $13.8 \pm 1.7$ years, the mean IQ was $104 \pm 12$ and their mean reading age was $11.7 \pm 1.5$ years. An age-matched control group of non-dyslexic children was recruited from the same college, their mean age was $12.3 \pm 1.8$ years; all had no known neurological or psychiatric abnormalities, no history of reading difficulty, no visual stress or any difficulties in near vision. IQ and reading measurements were not available for these children, but they were selected by the director of the school on the basis of their school performances; their scores in French (reading, understanding, orthography), mathematic and foreign languages were all beyond the mean score of the class. All children had normal binocular vision (60s of arc or better), which was evaluated with the TNO random dot test (Netherlands Organization of Applied Scientific Research Test of stereoacuity). Visual acuity was normal $(\geq 20 / 25)$ for all children. The investigation adhered to the principles of the Declaration of Helsinki, and was approved by our institutional human experimentation committee. Informed consent was obtained from children's parents after explanation of the procedure of the experiment.

\section{Oculomotor task}

The visual target was presented on a computer screen placed at $57 \mathrm{~cm}$ from the participant. Stimulus consisted of a black dot $\left(0.2^{0}\right)$ in the center of a grey background. For horizontal pursuit, after a fixation period $(1 \mathrm{~s})$ the black dot jumped $3^{0}$ either to the right or to the left and then moved in the opposite direction at a constant velocity of $15^{\circ} / \mathrm{s}$. It stopped when an eccentric position of $12^{0}$ was reached. For vertical pursuit the same paradigm was used with the initial target step, up or down, in the direction opposite to that of the smooth target motion. This step reduced the probability of occurrence of the first catch-up saccade during pursuit initiation (Rashbass, 1961). Each block for horizontal or vertical included 20 trials, 10 to each of the two directions, left/right or up/down, respectively, lasted about 3 minutes. The instruction given to the participant was to pursuit the moving dot as accurately as possible.

\section{Eye movement recording}

The Chronos Skalar video oculography apparatus was used. This system is based on infrared cameras using the CMOS technology (Clarke, Ditterich, Druen, Schonfeld $\&$ Steineke, 2002). Besides the cameras, the Chronossystem includes a number of hardware and software components that are all important for the overall system characteristics. During image acquisition (recording), the camera images are formatted in digital image frames. In this study, we selected the image sampling rates at $200 \mathrm{~Hz}$ for recording. The measurement resolution is better than $0.05^{\circ}$ and noise limits at $<0.02^{\circ}$.

\section{Data analysis}


CUS were defined as saccades occurring during pursuit, in the direction of target motion, which take the eyes from a position behind the target to one or near the target. Saccades that occurred after the start of target motion but prior to the start of pursuit were omitted; similar criteria have been used by others (Friedman, Jesberger, Abel \& Meltzer, 1992). Saccades were identified on the basis of velocity and acceleration criteria (eye velocity $>35 \%$; eye acceleration $>1000 \% \mathrm{~s}^{2}$, (Moschner, Crawford, Heide, Trillenberg, Kompf \& Kennard, 1999). Markers of CUS are shown in Fig.1 for recording in one non-dyslexic child. After removing the CUS, the amplitude and duration of remaining pursuit segment were analyzed. For each pursuit segment eye velocity ( $\mathrm{deg} / \mathrm{s})$ was determined as the ratio of amplitude to duration.
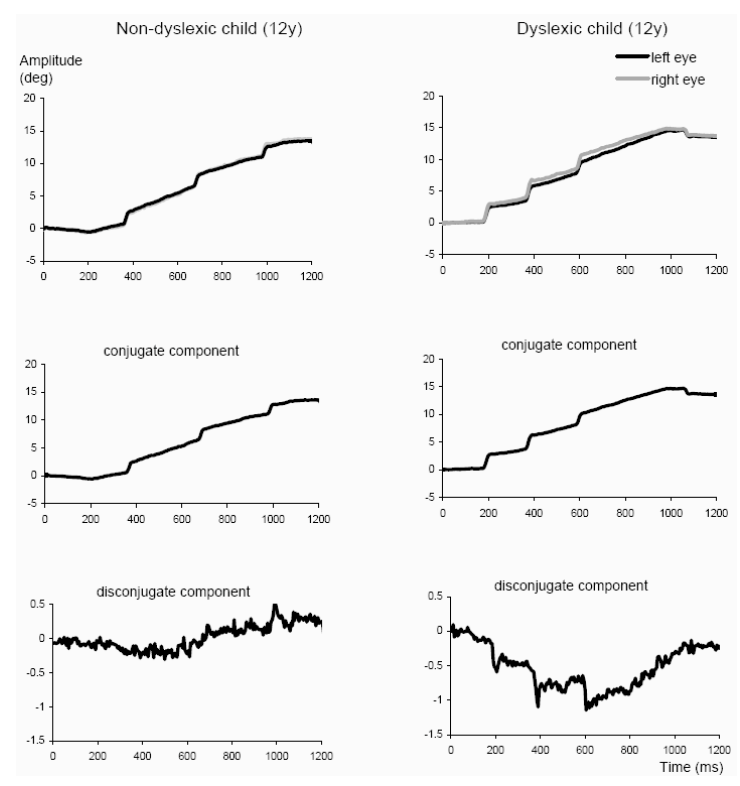

Figure 1. On the top, typical recordings of rightward smooth pursuit for both eyes from one non-dyslexic and one dyslexic; in the middle, conjugate component obtained by averaging the position signal of the two eyes $(L E+R E) / 2$; in the bottom, disconjugate component by the difference of position signal of the two eyes ( $L E-R E)$. Time $=0$ indicates the target presentation.

We measured the gain of pursuit, i.e. ratio of mean eye velocity of pursuit (without saccades) to the stimulus target velocity, the number and mean amplitude of CUS during pursuit, and the difference of amplitude between two eyes during pursuit smooth phases (pursuit disconjugacy) or during CUS (saccade disconjugacy, see Fig.1). The disconjugacy (left eye - right eye) could be positive or negative, i.e. convergent or divergent. Here, we used the absolute value for measuring the disconjugacy, i.e. the amplitude difference between two eyes. Absolute values are used for both saccade and pursuit disconjugacy measures.

The Mann-Whitney U test was used for comparisons between control group and dyslexic for each parameter. The Friedmann test was used to test the direction effect (left, right, up, down) for each parameter and for each group of participants. The Wilcoxon signed ranks test was used to compare results for any two directions; Bonferroni correction was made for multiple testing, and a corrected significance level of $\mathrm{p}<0.05$ was accepted.

\section{Results}

\section{Pursuit parameters}
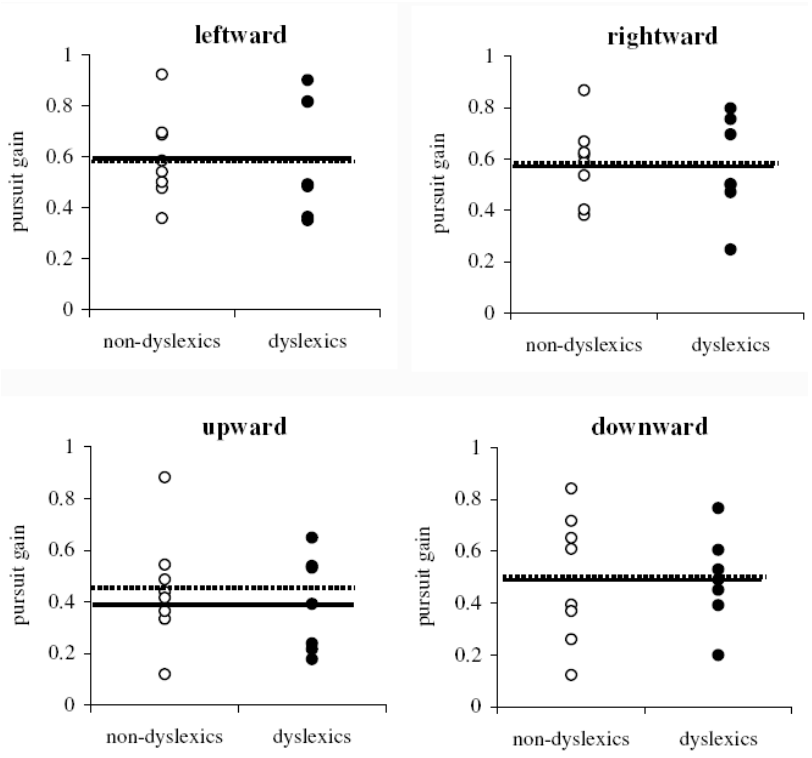

Figure 2. Individual mean gain of leftward, rightward, upward and downward pursuit for non-dyslexic and dyslexic children. The dashed and solid lines indicate the group mean for non-dyslexics and for dyslexics, respectively.

Figure 2 presents individual pursuit gain for non-dyslexic and dyslexic children for leftward, rightward, upward and downward pursuit. The Mann-Whitney U test comparing the gain of pursuit between non-dyslexic and dyslexic children showed no statistically significant difference for any direction (all $\mathrm{p}>0.05$ ). The Friedmann test applied on each group data on the gain of pursuit showed no direc- 
tion effect, neither for non-dyslexics $\left(\chi^{2}=4.2, \mathrm{p}=0.24\right)$ nor for dyslexics $\left(\chi^{2}=3.0, p=0.39\right)$.

Figure 3 presents individual number of CUS during pursuit for non-dyslexic and dyslexic groups for leftward, rightward, upward and downward pursuit. The MannWhitney U test comparing the number of CUS showed no statistically significant difference (for all directions $\mathrm{p}>0.05$ ) between non-dyslexics and dyslexics. The Friedmann test applied on each group data showed no significant direction effect for the number of CUS, neither for dyslexics $\left(\chi^{2}=5.85, \mathrm{p}=0.12\right)$ nor for non-dyslexics $\left(\chi^{2}=1.37, \mathrm{p}=0.71\right)$.
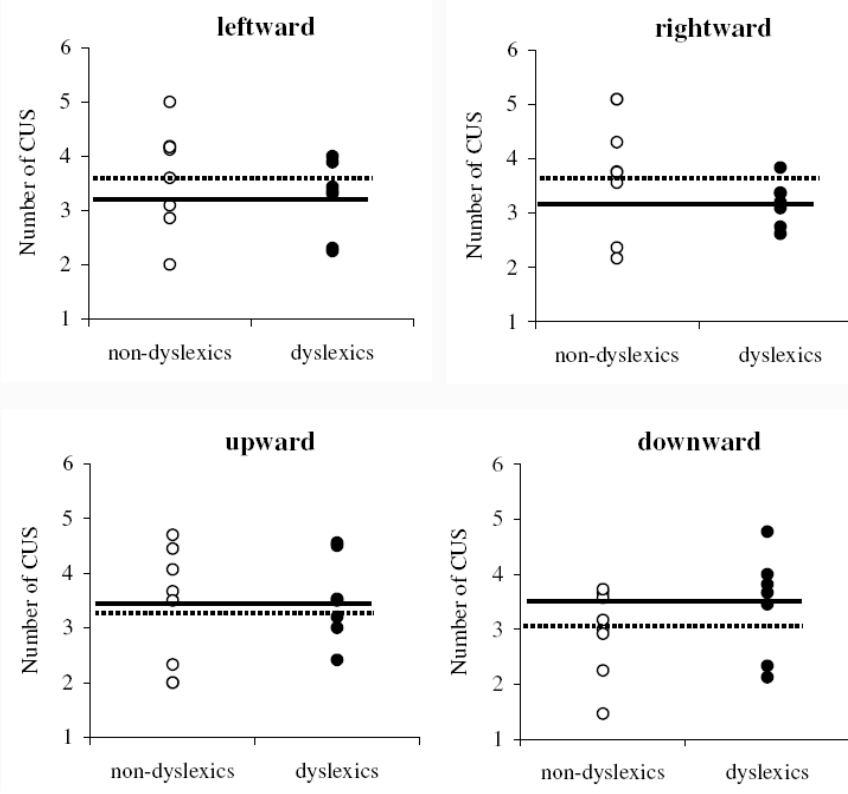

Figure 3. Number of catch-up saccades (CUS) for each participant during leftward, rightward, upward and downward pursuit for non-dyslexic and dyslexic children. Other notations as in Fig.2.

Figure 4 presents individual amplitude of CUS during pursuit for non-dyslexic and dyslexic groups for leftward, rightward, upward and downward pursuit. The MannWhitney U test comparing the amplitude of CUS between dyslexics and non-dyslexics showed no statistically significant difference (for all directions $\mathrm{p}>0.05$ ). The Friedmann test applied on each group data showed a direction effect for non-dyslexics only $\left(\chi^{2}=17.25, \mathrm{p}<0.001\right)$ : lower mean amplitude of CUS for horizontal than for vertical pursuit (all comparisons $\mathrm{p}<0.012$ ), i.e. left versus up or down, right versus up or down.
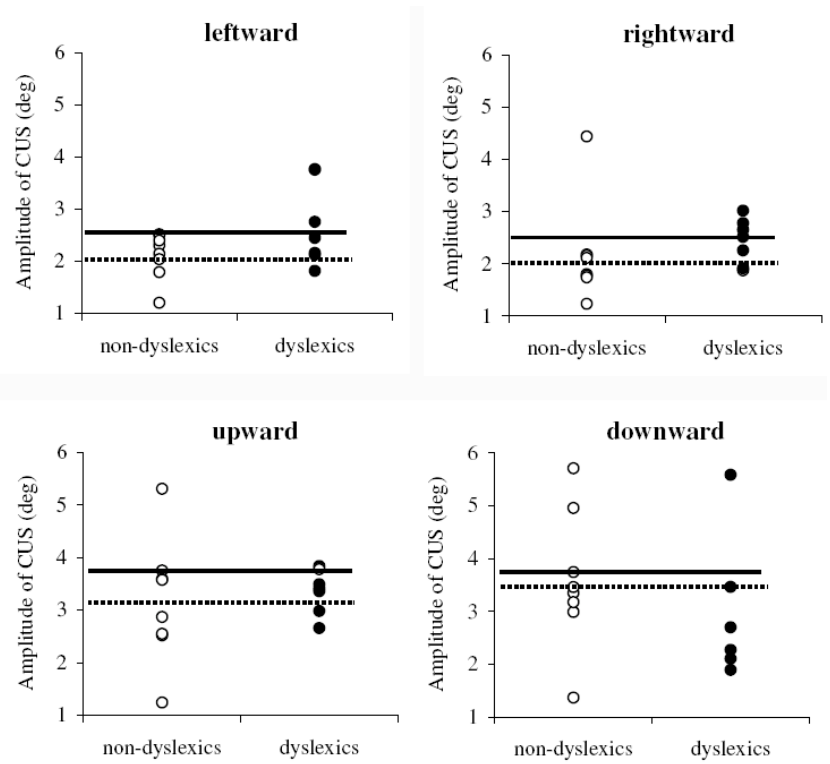

Figure 4. Individual mean amplitude of catch-up saccades (CUS) during leftward, rightward, upward and downward pursuit for non-dyslexic and dyslexic children. Other notations as inFig.2.

\section{Disconjugacy values}

Figure 5 presents individual disconjugacy (absolute values) for smooth phase of pursuit for non-dyslexic and dyslexic groups in all directions (left, right, up and down). The Mann-Whitney $U$ test showed significantly higher disconjugacy of smooth phase in non-dyslexics than in dyslexics for rightward pursuit only ( $U=9$, $\mathrm{p}<0.05)$. The Friedmann test applied on each group data for the disconjugacy for smooth phase of pursuit showed a direction effect for non-dyslexics only $\left(\chi^{2}=11.85\right.$, $\mathrm{p}<0.01)$ : the disconjugacy amplitude of pursuit to the right was significantly lower than for pursuit to the down $(\mathrm{p}<0.012)$.

Figure 6 presents individual disconjugacy (absolute values) for CUS during pursuit for non-dyslexic and dyslexic groups in all directions (left, right, up and down).

The Mann-Whitney $U$ test showed significantly higher disconjugacy of CUS in dyslexics than in non-dyslexics for rightward pursuit only $(U=9, p<0.05)$. There was a direction effect for both groups of participants $\left(\chi^{2}=10.05\right.$, $\mathrm{p}<0.01$ for non-dyslexics; $\chi^{2}=8.8, \mathrm{p}<0.05$ for dyslexics). Comparisons between any two directions with the Wilcoxon test showed for non-dyslexics lower amplitude of saccade disconjugacy for pursuit to the right than that to the left or to the down (both $\mathrm{p}<0.012$ ); for dyslexics 
lower amplitude of the saccade disconjugacy for pursuit to the left than that to the right $(\mathrm{p}<0.012)$.
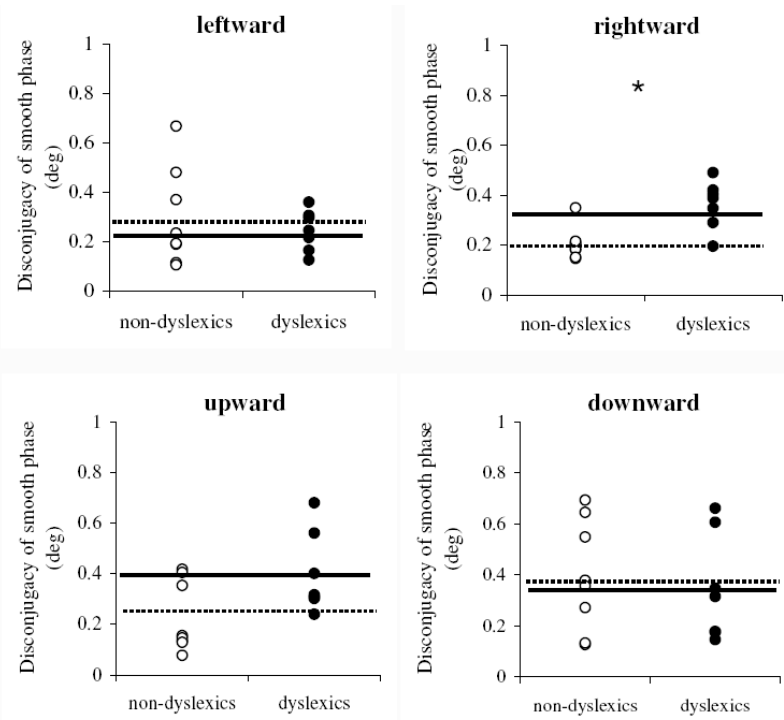

Figure 5. Individual means of disconjugacy of a smooth phase of leftward, rightward, upward and downward pursuit for non-dyslexic and dyslexic children. Asterisk indicates the difference between two groups. Other notations as in Fig. 2.
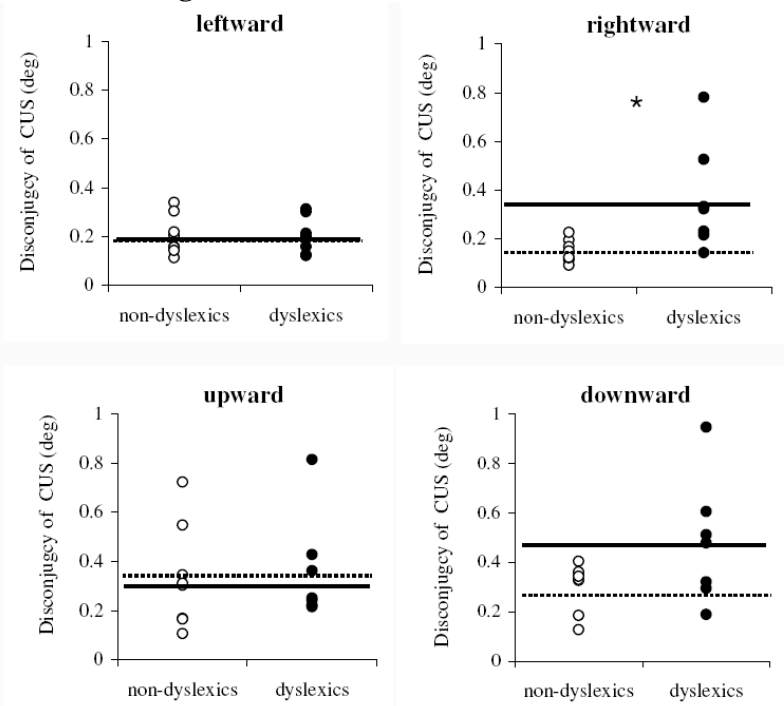

Figure 6. Individual means of disconjugacy of CUS during leftward, rightward, upward and downward pursuit for non-dyslexic and dyslexic children. Other notations as in Fig. 5.

In summary, the data show no group effect for the gain of SP, number of CUS or the amplitude of CUS. They show a group effect on disconjugacy only, i.e. higher disconjugacy of both smooth phase of pursuit and of CUS for dyslexics but this occurs only for rightward pursuit.

\section{Discussion}

Similar characteristics of SP in normal and dyslexic children

Our results show that the gain of pursuit, the number and amplitude of CUS are similar for dyslexic and non-dyslexic children. These results are not compatible with the studies by Black et al. (1984) and Eden et al. (1994), who reported abnormal smooth pursuit with increased number of saccadic intrusions in dyslexics. Snashall (1983) also reported smooth pursuit ataxia in reading disabled children. However, these studies reported that only $16 \%-25 \%$ of dyslexic children had abnormal CUS or SP ataxia. When SP is insufficient to track a moving target, CUS are employed to capture it. CUS could be more frequent during pursuit tasks for various reasons including stimulus parameters - the target step and ramp (de Brouwer, Yuksel, Blohm, Missal \& Lefevre, 2002), fatigue effect (Judge, Caravolas \& Knox, 2006), age (Fukushima, Tanaka, Williams \& Fukushima, 2005) and pathology, e.g. psychotic disorders (Kumra, Sporn, Hommer, Nicolson, Thaker, Israel, Lenane, Bedwell, Jacobsen, Gochman \& Rapoport, 2001), autism (Takarae, Minshew, Luna, Krisky \& Sweeney, 2004), childhood-onset schizophrenia (Randall \& Barroso, 2002). For non-dyslexics there are conflicting reports with regard to the age at which the smooth pursuit eye movement system children with learning disorders (from the 18 studied children with 5 reading disorders). Langaas et al. (1998) reported horizontal SP gain of 0.97 in children aged 5-7 years when they tracked a sinusoidally moving target at $0.3 \mathrm{~Hz}$ frequency. However, many studies reported lower SP gain in children in comparison to adults. Katsanis, Iacono \& Harris (1998) used infrared eye tracker to measure SP of a sinusoidally moving target in 137 participants aged 11-12 years, 17-18 years and adults. Horizontal SP gains were reported to reach adult values at 17-18 years. Ross, Radant \& Hommer (1993) reported horizontal SP gain of 0.88 in 53 school-age children for a target moving at a constant velocity of $12 \%$ s (lower than adults). Similar SP gain values for children and gain increased with age have been reported in other studies (Jacobsen, Hong, Hommer, Hamburger, Castellanos, Frazier, Giedd, Gordon, Karp, McKenna \& Rapoport, 1996). The development of SP with age reflects ongoing brain development in children. Lower SP gain in children may reflect the immaturity of several processes that occur during computation of the target and eye movement velocities which involves translation from 
sensory to motor coordinates, speed of sensory processing of motion, motivation or attention (Jacobs, Harris, Shawkat \& Taylor, 1997). Development maturation is related to brain myelination, which progresses from dorsal to ventral brain regions. Frontal, temporal and posterior parietal cortex, which are involved in SP processing (Leigh \& Zee, 2006), continue to acquire myelin throughout childhood and early adulthood (Barkovich, 2000). Such development of related cortex in control of SP occurs for both dyslexics and non-dyslexics; it may be difficult to differentiate some hidden SP gain problems in dyslexics within these overall low gains for pursuit. .

\section{Direction specific pursuit disconjugacy in dyslexics}

The new finding of this study is that dyslexic children showed poorer binocular coordination during SP but only for rightward stimulation. A few studies of saccades and fixations have shown binocular abnormality in dyslexics (Bucci et al., 2008b, Kapoula, Bucci, Jurion, Ayoun, Afkhami \& Bremond-Gignac, 2007). Eden et al. (1994) reported fixation instability and lower vergence amplitudes in dyslexic children. Stein et al. (2000) reported that $64 \%$ of dyslexic children were unable to make proper vergence movements when macular sized fusion targets $\left(2.5^{0}\right)$ were employed. Kapoula et al. (2007) also reported abnormal vergence in dyslexic children, e.g. the near point of convergence was significantly more remote in dyslexics; divergence at both far and near was significantly reduced in dyslexics. Bucci et al. (2008b) reported poor binocular coordination during saccades and fixations for dyslexic children. Kapoula et al. (2008) extended further these observations for saccades during free exploration of paintings. The ensemble of the studies indicates an intrinsic ocular motor deficiency, which could be related to immaturity of the normal ocular learning mechanisms via which binocular coordination and stable fixation are achieved (Bucci et al., 2008b); as proposed by Kapoula et al.(2008) proper function of the veregnce eye movement control system could be important for binocular coordination of saccades and stable fixation. Our results extend observations for weak binocular coordination for smooth phase of pursuit and the CUS.

An important aspect is the direction specific deficit. Inspection of the individual pursuit results shows that for the rightward direction, individual mean values of disconjugacy and intersubject variability (standard deviation/mean) are particularly small for non-dyslexics (about $30 \%$ for rightward $v s$ about $60 \%$ for other directions, see figures 5 and 6). Perhaps due to left to right reading training pursuit in this direction becomes highly skilful and optimized relative to other directions even though statistically significant difference was observed. If binocular coordination for rightward direction is optimized because of reading then one should expect optimal binocular coordination for saccades as well. Such optimization remains to be searched by comparing small size rightward readings saccades and leftward reading saccades. Bucci et al. (2008b) and Kapoula et al. (2008) studied large size saccades $\left(>5.4^{\circ}\right)$ and poor binocular coordination appeared for both left and right directions in dyslexics. Binocular coordination of small size saccades such as during reading in dyslexics remains to be investigated. In the pursuit study the difference for this pursuit direction only could simply mean that such optimization has not yet been achieved due to insufficiency of underlying visuo-attention mechanisms. On overall, one can conclude that binocular control during smooth pursuit in dyslexics similarly to non-dyslexics is of good quality; nevertheless, the degree of optimization for rightward pursuit achieved by non-dyslexics is not seen in dyslexics. Further experiments on subjects trained with right-toleft reading (Hebrew or Arabic) are of interest to explore the hypothesis of optimization to reading practice.

Problems in smooth pursuit generally are not linked to specific neurological and/or cognitive deficits; poor binocular coordination during rightward pursuit in dyslexics perhaps could be a manifestation of delayed development of binocular learning mechanisms that are known to depend on posterior parietal function (Vernet et al., 2008) and cerebellum (Versino, Hurko \& Zee, 1996); both these areas directly or indirectly receive massive input from magnocellular pathways. However, this result does not exclude problems on phonological development in dyslexics. As pointed by (Pernet, Andersson, Paulesu \& Demonet (2009) the main theories, Phnological, magnocellular - visual and /or auditory and cerebellar (Ramus et al., 2003) for dyslexia are not mutually exclusive, each emphasizes the importance of a given aspect only. For example, the visual theory does not exclude a phonological deficit, but emphasizes a visual contribution to reading problems, at least in some dyslexic individuals. Moreover, phonological awareness is strongly related to reading ability. Callu, Giannopulu, Escolano, Cusin, Jacquier-Roux \& Dellatolas (2005) assessed clinically pursuit eye movements; they reported that children who failed at smooth pursuit showed lower scores at a number of cognitive tasks, especially phonological awareness tasks, and copy of visually presented trajectories. They thought that perhaps frontal cortex immaturity may explain these associations in preschool children. Failure to optimize binocular coordination of pursuit for the right direction in dyslexics could be related to decreased visual function 
of magnocellular and/or cerebellar areas, which are also involved in reading itself.

In conclusion, dyslexic children studied here do not show impaired pursuit relative to non-dyslexic; gain of the pursuit is, however, low particularly in the vertical direction and CUS are frequent for both populations. The quality of binocular coordination is also good for both groups. For non-dyslexics for smooth pursuit to the right the quality of binocular coordination is particularly good, but this privilege hypothetically related to training via reading, does not appear for dyslexics. Even though mild and direction specific, the reduced quality of the binocular coordination during smooth pursuit to the right adds to previous studies of saccades and suggests immaturity of oculomotor learning mechanisms in dyslexics. These observations, however, need to be confirmed in a larger population including older children and compared with other populations, for example, with right-to-left reading.

\section{References}

Barkovich, A.J. (2000). Concepts of myelin and myelination in neuroradiology. AJNR Am J Neuroradiol, 21 (6), 1099-1109.

Biscaldi, M., Fischer, B., \& Aiple, F. (1994). Saccadic eye movements of dyslexic and normal reading children. Perception, 23 (1), 45-64.

Biscaldi, M., Gezeck, S., \& Stuhr, V. (1998). Poor saccadic control correlates with dyslexia. Neuropsychologia, 36 (11), 1189-1202.

Black, J.L., Collins, D.W., De Roach, J.N., \& Zubrick, S.R. (1984). Smooth pursuit eye movements in normal and dyslexic children. Percept Mot Skills, 59 (1), 91-100.

Bucci, M.P., Bremond-Gignac, D., \& Kapoula, Z. (2008a). Latency of saccades and vergence eye movements in dyslexic children. Exp Brain Res, 188 (1), 1-12.

Bucci, M.P., Bremond-Gignac, D., \& Kapoula, Z. (2008b). Poor binocular coordination of saccades in dyslexic children. Graefes Arch Clin Exp Ophthalmol, 246 (3), 417-428.

Callu, D., Giannopulu, I., Escolano, S., Cusin, F., Jacquier-Roux, M., \& Dellatolas, G. (2005). Smooth pursuit eye movements are associated with phonological awareness in preschool children. Brain Cogn, 58 (2), 217-225.

Chevrie-Muller, C., Simon, A.M., \& Fournier, S. (1997). Batterie language oral écrit, mémoire, attention (L2MA). (Paris: Editions du centre de psychologie appliquée.
Clarke, A.H., Ditterich, J., Druen, K., Schonfeld, U., \& Steineke, C. (2002). Using high frame rate CMOS sensors for three-dimensional eye tracking. Behav Res Methods Instrum Comput, 34 (4), 549-560.

Cornelissen, P., Bradley, L., Fowler, S., \& Stein, J. (1991). What children see affects how they read. Dev Med Child Neurol, 33 (9), 755-762.

Cornelissen, P.L., Hansen, P.C., Hutton, J.L., Evangelinou, V., \& Stein, J.F. (1998). Magnocellular visual function and children's single word reading. Vision Res, 38 (3), 471-482.

de Brouwer, S., Yuksel, D., Blohm, G., Missal, M., \& Lefevre, P. (2002). What triggers catch-up saccades during visual tracking? J Neurophysiol, 87 (3), 16461650.

Eden, G.F., Stein, J.F., Wood, H.M., \& Wood, F.B. (1994). Differences in eye movements and reading problems in dyslexic and normal children. Vision Res, 34 (10), 1345-1358.

Friedman, L., Jesberger, J.A., Abel, L.A., \& Meltzer, H.Y. (1992). Catch-up saccade amplitude is related to square wave jerk rate. Invest Ophthalmol Vis Sci, 33 (1), 228-233.

Fukushima, J., Tanaka, S., Williams, J.D., \& Fukushima, K. (2005). Voluntary control of saccadic and smoothpursuit eye movements in children with learning disorders. Brain Dev, 27 (8), 579-588.

Jacobs, M., Harris, C.M., Shawkat, F., \& Taylor, D. (1997). Smooth pursuit development in infants. Aust N Z J Ophthalmol, 25 (3), 199-206.

Jacobsen, L.K., Hong, W.L., Hommer, D.W., Hamburger, S.D., Castellanos, F.X., Frazier, J.A., Giedd, J.N., Gordon, C.T., Karp, B.I., McKenna, K., \& Rapoport, J.L. (1996). Smooth pursuit eye movements in childhood-onset schizophrenia: comparison with attention-deficit hyperactivity disorder and normal controls. Biol Psychiatry, 40 (11), 1144-1154.

Judge, J., Caravolas, M., \& Knox, P.C. (2006). Smooth pursuit eye movements and phonological processing in adults with dyslexia. Cognitive neuropsychology, 23 (8), 1174-1189.

Kapoula, Z., Bucci, M.P., Jurion, F., Ayoun, J., Afkhami, F., \& Bremond-Gignac, D. (2007). Evidence for frequent divergence impairment in French dyslexic children: deficit of convergence relaxation or of divergence per se? Graefes Arch Clin Exp Ophthalmol, 245 (7), 931-936.

Kapoula, Z., Ganem, R., Poncet, S., Gintautas, D., Eggert, T., Bremond-Gignac, D., \& Bucci, M.P. (2008). Free exploration of painting uncovers particularly loose yoking of saccades in dyslexics. Dyslexia,

Katsanis, J., Iacono, W.G., \& Harris, M. (1998). Development of oculomotor functioning in preadolescence, 
adolescence, and adulthood. Psychophysiology, 35 (1), 64-72.

Krauzlis, R.J., Basso, M.A., \& Wurtz, R.H. (2000). Discharge properties of neurons in the rostral superior colliculus of the monkey during smooth-pursuit eye movements. J Neurophysiol, 84 (2), 876-891.

Kumra, S., Sporn, A., Hommer, D.W., Nicolson, R., Thaker, G., Israel, E., Lenane, M., Bedwell, J., Jacobsen, L.K., Gochman, P., \& Rapoport, J.L. (2001). Smooth pursuit eye-tracking impairment in childhood-onset psychotic disorders. Am J Psychiatry, 158 (8), 1291-1298.

Langaas, T., Mon-Williams, M., Wann, J.P., Pascal, E., \& Thompson, C. (1998). Eye movements, prematurity and developmental co-ordination disorder. Vision Res, 38 (12), 1817-1826.

Leigh, R.J., \& Zee, D.S. (2006). The neurology of eye movements (fourth edition). (pp. 189-191). New-York Oxford: Oxford University Press.

Moschner, C., Crawford, T.J., Heide, W., Trillenberg, P., Kompf, D., \& Kennard, C. (1999). Deficits of smooth pursuit initiation in patients with degenerative cerebellar lesions. Brain, 122 ( Pt 11), 2147-2158.

Nicolson, R.I., Fawcett, A.J., \& Dean, P. (2001). Developmental dyslexia: the cerebellar deficit hypothesis. Trends Neurosci, 24 (9), 508-511.

Pavlidis, G.T. (1981). Do eye movements hold the key to dyslexia? Neuropsychologia, 19 (1), 57-64.

Pernet, C., Andersson, J., Paulesu, E., \& Demonet, J.F. (2009). When all hypotheses are right: A multifocal account of dyslexia. Hum Brain Mapp,

Rae, C., Karmiloff-Smith, A., Lee, M.A., Dixon, R.M., Grant, J., Blamire, A.M., Thompson, C.H., Styles, P., \& Radda, G.K. (1998). Brain biochemistry in Williams syndrome: evidence for a role of the cerebellum in cognition? Neurology, 51 (1), 33-40.

Ramus, F., Rosen, S., Dakin, S.C., Day, B.L., Castellote, J.M., White, S., \& Frith, U. (2003). Theories of developmental dyslexia: insights from a multiple case study of dyslexic adults. Brain, $126(\mathrm{Pt} 4), 841-865$.

Randall, M., \& Barroso, J. (2002). Delayed pursuit of health care among HIV-positive gay men enrolled in a longitudinal research study. $J$ Assoc Nurses AIDS Care, 13 (4), 23-31.

Rashbass, C. (1961). The relationship between saccadic and smooth tracking eye movements. J Physiol, 159, 326-338.

Ross, R.G., Radant, A.D., \& Hommer, D.W. (1993). A developmental study of smooth pursuit eye movements in normal children from 7 to 15 years of age. $J$ Am Acad Child Adolesc Psychiatry, 32 (4), 783-791.

Snashall, S.E. (1983). Vestibular function tests in children. J R Soc Med, 76 (7), 555-559.
Sparks, D.L., \& Jay, M.F. (1986). The functional organization of the primate superior colliculus: a motor perspective. Prog Brain Res, 64, 235-241.

Stein, J. (2001). The magnocellular theory of developmental dyslexia. Dyslexia, 7 (1), 12-36.

Stein, J.F., Richardson, A.J., \& Fowler, M.S. (2000). Monocular occlusion can improve binocular control and reading in dyslexics. Brain, 123 ( Pt 1), 164-170.

Stein, J.F., Riddell, P.M., \& Fowler, S. (1988). Disordered vergence control in dyslexic children. Br JOphthalmol, 72 (3), 162-166.

Takagi, M., Zee, D.S., \& Tamargo, R.J. (2000). Effects of lesions of the oculomotor cerebellar vermis on eye movements in primate: smooth pursuit. J Neurophysiol, 83 (4), 2047-2062.

Takarae, Y., Minshew, N.J., Luna, B., Krisky, C.M., \& Sweeney, J.A. (2004). Pursuit eye movement deficits in autism. Brain, 127 (Pt 12), 2584-2594.

Tian, J.R., \& Lynch, J.C. (1996). Functionally defined smooth and saccadic eye movement subregions in the frontal eye field of Cebus monkeys. $J$ Neurophysiol, 76 (4), 2740-2753.

Vernet, M., Yang, Q., Daunys, G., Orssaud, C., Eggert, T., \& Kapoula, Z. (2008). How the brain obeys Hering's law: a TMS study of the posterior parietal cortex. Invest Ophthalmol Vis Sci, 49 (1), 230-237.

Versino, M., Hurko, O., \& Zee, D.S. (1996). Disorders of binocular control of eye movements in patients with cerebellar dysfunction. Brain, 119 ( Pt 6), 1933-1950. 